\title{
Planetary Nomenclature and Indigenous Communities
}

\author{
A white paper to be submitted to the \\ Planetary Science and Astrobiology Decadal Survey 2023 \\ 15 September 2020
}

\begin{abstract}
Authors:
Matthew S. Tiscareno, SETI Institute

Daniella M. Scalice, NASA Ames Research Center

Meagan L. Thompson, NASA Headquarters

Jessica L. Noviello, Arizona State University

Vivian White, Astronomical Society of the Pacific

Aparna Venkatesan, University of San Francisco

Moses Milazzo, Other Orb LLC

Kat Gardner-Vandy (Choctaw Nation of Oklahoma), Oklahoma State University

Sarah EchoHawk (enrolled member of the Pawnee Nation of Oklahoma), American Indian

Science and Engineering Society

Michael Laverdure (Migisi Migwan, Makwa Dodem, citizen of the Turtle Mountain Band of

Chippewa), American Indian Science and Engineering Society

Crystal Echo Hawk (enrolled member of the Pawnee Nation of Oklahoma), IllumiNatives

Larry Kimura, Ka Haka 'Ula O Ke 'elikōlani College of Hawaiian Language, University of

Hawai'i at Hilo

Doug Simons, Canada-France-Hawaii Telescope

Alan Tokunaga, Institute for Astronomy, University of Hawai'i

Ka'iu Kimura, 'Imiloa Astronomy Center of Hawai'i, University of Hawai'i at Hilo

Michael Waasegiizhig Price (Anishinaabe and tribal member of Wikwemikong First Nations), Great Lakes Indian Fish and Wildlife Commission
\end{abstract}

This white paper is submitted as part of a collaborative effort organized by the Equity, Diversity, and Inclusion Working Group (EDIWG), a cross Assessment Group (AG) committee.

A list of supporters can be found at https://seti.org/PNIC-WP20_supporters (link)

To sign on as a supporter, please email matt@seti.org 
Executive Summary: In recent decades, planetary nomenclature has expanded from drawing almost exclusively from European cultures to become more culturally diverse. However, Indigenous communities have generally not been included in decisions to use their cultural property for planetary nomenclature. Actively including Indigenous voices in naming decisions ensures that their cultures are accurately and respectfully represented and recognizes their sovereignty over their own culture. The planetary community and NASA should build strong co-creative relationships with Indigenous communities, eventually resulting in procedures for responsible use of cultural property in planetary nomenclature.

\section{Background}

\subsection{Stage One: Euro-centrism}

The five planets visible to the naked eye have many names in many cultures, though Western astronomers generally use the names that were applied to them by the Romans (Mercury, Venus, Mars, Jupiter, and Saturn). Although the first planetary moons were discovered in the 1600s, it was not until the 1800s that consensus was reached on their names, which for all planets and their moons until 2000 continued to be drawn exclusively from Greco-Roman mythology or (in the anomalous case of the Uranian moons) English literature.

Around the same time, the first asteroids were given names. The first several dozen again came mostly from Greco-Roman mythology, though by the 1850 s it became established that the discoverer of an asteroid could name it in nearly any way they wanted. This still resulted in names drawn almost entirely from Euro-American culture, due to global inequities by which asteroid discoverers themselves came predominantly from such cultures. Similarly, comets are named directly after their discoverers, leading to analogous patterns.

Features on planetary surfaces also receive names, the earliest being those on the Moon. In the early 1900s, the newly formed International Astronomical Union (IAU) established itself as the consensus arbiter for basins, craters, and other topographic features on the Moon. ${ }^{1}$ In 1958, the IAU began to play a similar role for features on the surface of Mars. ${ }^{2}$ At this point, all names were still drawn from European cultures.

\subsection{Stage Two: Towards multiculturalism}

In the 1970s, the IAU began to look beyond Euro-centric cultures, introducing a focus on "worldwide representation" in the names of planetary surface features. ${ }^{3}$ It is evident that this shift was borne at least partly from a recognition that the previous Euro-centric scope of planetary names inadequately represented the breadth of global participation in science. However, despite a more global scope in selected names, nearly all of the individuals participating in the actual decision-making process were from North America or Europe (a few were from China or Japan). ${ }^{4}$

\footnotetext{
${ }^{1}$ Gaither T and Hayward R (2018). Planetary Nomenclature: A Brief History and Overview. LPI Bulletin 151, 1-9. Available at https://www.Ipi.usra.edu/publications/newsletters/lpib/new/planetarynomenclature ${ }^{2}$ Dollfus A (1958). Nomenclature martienne selon I'Union Astronomique Internationale. L'Astronomie 72, 453 (link).

${ }^{3}$ Hargitai $\mathrm{H}$ and Nass A (2019). Planetary Mapping: A Historical Overview. In Hargitai H (ed.), Planetary Cartography and GIS (Springer), 27-64.

${ }^{4}$ Masursky H et al. (1986). Gazetteer of planetary nomenclature. U.S. Geological Survey Open File Report 84-692. Available at https://pubs.er.usgs.gov/publication/ofr84692
} 
In 2000, the first names drawn from Inuit culture were given to a class of small distant moons of Saturn by a Western astronomer who worked with an Inuit author to develop the names. ${ }^{5}$ There are now five named "Inuit" moons (Kiviuq, Ijiraq, Paaliaq, Siarnaq, and Tarqeq), which remain the only planetary ${ }^{6}$ moons to bear non-European names.

\subsection{Cultural appropriation and the spirit of colonialism}

While the expansion of planetary nomenclature to honor more global cultures is commendable, it has also commonly been marked by cultural appropriation. Cultural appropriation occurs when people from one culture use or adopt the cultural property ${ }^{7}$ of another culture, but it is problematic especially when a dominant culture unilaterally takes from an Indigenous culture without consent.

Cultural appropriation is an aspect of colonialism, which is the posture of any culture that seeks to exercise domination over other cultures (the targets often being Indigenous cultures in their own ancestral lands). A related term in the U.S. context is "manifest destiny."

Dispossession of land and resources is a hallmark of colonialism, and such dispossession is still with us in the form of the many inequalities that exist within the United States and throughout the globe. However, the spirit of colonialism is also widely found in a sense of entitlement to pick and choose the elements of other cultures that one finds most enjoyable, and to enjoy that cultural property on one's own terms, apart from the people whose cultures created them.

The spirit of colonialism can co-exist with a genuine desire to honor other cultures. Our focus is not on intentions, but on actions and their effects. To truly respect others requires attentive listening, and that is precisely what is often lacking in Western relations with Indigenous communities. We say here that cultural appropriation and colonialism have been present in the trend towards a multicultural scope of planetary nomenclature because the decisions to apply specific names from global cultures have been made almost entirely by people from Euro-American cultures. This is not to deny the good intentions of those who have made such decisions, and we acknowledge that many Western astronomers have undertaken nomenclature tasks with little funding and with no relational resources in place. Our purpose here is rather to promote hearing of Indigenous voices with regard to the effects these developments have had on their communities, and to consider a better way.

\subsection{The need for Stage Three: Co-Creation and cultural self-determination}

The best way to honor a culture is to make space for the practitioners of that culture to speak for themselves. This has been infrequently practiced in planetary nomenclature to date. A spirit of co-creation would include Indigenous people from beginning to end of the decision-making process, giving them control over how their cultural heritage is to be used. Such co-creation cannot be summoned immediately when Westerners feel an Indigenous partner is needed and then filed away again when the "need" is past. Rather, it requires a foundation of intentional long-term relationships in which Western and Indigenous peoples collaborate on equal terms towards mutual goals. ${ }^{8}$ Moreover, the conveyance of a name is a

\footnotetext{
${ }^{5}$ Petten C (2003). Naming Saturn's moons. Windspeaker 21 (7), 32-33 (link) (link).

${ }^{6}$ A number of minor-planet moons have received non-European names in the past 15 years.

${ }^{7}$ Although the term "cultural property" may imply concepts of ownership or possession that are foreign to most Indigenous cultures, we use it in the sense of heritage that is an attribute of a particular community.

${ }^{8}$ Gardner-Vandy K et al. (2020). Relationship First and Always: A Guide to Collaborations with Indigenous Communities. White paper submitted to the Planetary Science and Astrobiology Decadal Survey 2023 (link).
} 
sacred practice in nearly all Indigenous cultures worldwide, ${ }^{9}$ as Indigenous Knowledge is deeply rooted in the traditional language base of each community. Conferring a name in the absence of ceremony, as much as in the absence of consent, is symptomatic of cultural appropriation.

We cannot avoid the need for planetary nomenclature, because objects and features in the solar system are constantly being discovered and it is right and prudent to name them. However, the scientific community must no longer feel entitled to appropriate cultural property without consent and participation, which perpetuates the spirit of colonialism. The only tenable way forward is to co-create, giving true agency to Indigenous scientists as well as Indigenous cultural practitioners alongside Western astronomers, so that the scientific enterprise might truly be for the benefit of all humankind.

\section{Native American Communities}

The problems of cultural appropriation and colonialism, and the remedies of relationship-building and co-creation, apply to Indigenous cultures around the world. However, since this paper is written in the context of the United States of America, we here focus particularly on the Indigenous peoples of the U.S.

Modern Native Americans comprise some 1.7\% of the U.S. population, but they occupy a far smaller percentage of the consciousness of most Americans. According to a recent study, ${ }^{10} 87 \%$ of K-12 schools in the U.S. do not teach about Native Americans past 1900, thus keeping them relegated to the past in the consciousness of many. Furthermore, representation of Native Americans in television and film is just $0.04 \%$, and many of these are in historical settings prior to 1900 and heavily reliant on stereotypes such as the "mystical Indian" or the "noble warrior." As a result, $78 \%$ of Americans know little to nothing about the identities, values and successes of modern Native Americans, or the issues they face. Co-creation of planetary nomenclature would help address this problem by actively including and highlighting the contributions of modern Indigenous people in science and engineering.

Native Americans are under-represented in the planetary workforce by $42 \% \pm 38 \%$ (with a two-sigma error bar) relative to the national workforce, which is substantial though error bars are high due to low numbers overall, ${ }^{11}$ despite efforts in recent decades to boost STEM education on reservations and in Native American communities. One reason for continued reluctance of Native American youth to participate in Western science careers is the continued lack of true relationship between their communities and White-dominated institutions. They often perceive a colonialist attitude of "ask, take, leave," as others arrive with an agenda already in place, obtain what they want from the Native American community, and then conclude the relationship. ${ }^{8}$ They also often perceive a disconnect between their Indigenous identity and the Western establishment, as if part of themselves must remain hidden in order to "fit in" with Western scientific culture.

From where most Native American languages stand, people may consider indulging in planetary nomenclature to have minuscule effect, yet such engagement can lead to much bigger ramifications. Many Indigenous communities are revitalizing their ancestral languages

\footnotetext{
9 Thornton TF (1997), Anthropological Studies of Native American Place Naming, American Indian Quarterly 21 (2), 209-228. Available at http://www.jstor.org/stable/1185645

${ }^{10}$ See the Reclaiming Native Truth survey, https://illuminatives.org/reclaiming-native-truth

${ }^{11}$ Rivera-Valentin EG et al. (2020). "Who is missing in planetary science?": A demographic study of the planetary science workforce. White paper submitted to the Planetary Science and Astrobiology Decadal Survey 2023 (link).
} 
and cultures, including their knowledge of the stars and other celestial phenomena that contain stories, values, and cultural practices. Co-creation of planetary nomenclature would bolster this revitalization and reduce disconnection, thus making space for Indigenous youth to engage with planetary science while remaining true to their whole person. ${ }^{12,13}$

Indigenous scholars from ${ }^{14}$ the American Indian Science and Engineering Society (AISES), the Society for Advancement of Chicanos/Hispanics and Native Americans in Science (SACNAS), the Indigenous Education Institute (IEI), the Smithsonian National Museum of the American Indian (NMAI), tribally controlled community colleges and universities (TCUs), the think tank IllumiNatives, and the 'Imiloa Astronomy Center of Hawai'i (some of whom are co-authors of this paper), among others, combine expertise in Western astronomy with expertise in traditional Indigenous Knowledge and in working with Indigenous communities, and stand ready to facilitate more effective partnerships with NASA and other institutions. Such partnerships would both enable the co-creation of planetary nomenclature and be further enabled by it.

\section{Lessons Learned from Recent Nomenclature Experiences}

\subsection{The NameExoWorlds project}

The International Astronomical Union (IAU) launched the NameExoWorlds project in 2019, giving 113 participating countries the chance to name an exoplanet and star visible from their country. ${ }^{15}$ One of the Naming Rules stated, "In recognition of the UN 2019 International Year of Indigenous Languages (IYIL2019), speakers of Indigenous languages are encouraged to propose names drawn from those languages." However, in the most recent report from the Working Group on Star Names, there is no mention of cultural sensitivity in using Indigenous naming. ${ }^{16}$ In the U.S., a small diverse team of astronomers and educators formed to lead this project. After collecting hundreds of names from the public, a committee of 40 reviewers chose the top 10 qualifying names and released those to the public for voting. Eight of the 10 names that made the final list were related to Native Americans and other Indigenous cultures, including Indigenous names of natural wonders, names of sun-gods, and traditional names for "star."

The U.S. organizing committee belatedly recognized the issues related to cultural appropriation. They began reaching out to leaders of Indigenous communities and Native astronomers for guidance and received good advice about how to proceed. Working to a tight timeline as dictated by the IAU, the U.S. committee chose its top 3 name pairs for the star and its exoplanet, two of which were of Indigenous origin. The top contender was successfully vetted as having been submitted by a young woman from the Curyung Tribal Council, referencing the names of two rivers in Alaska that are important to the Indigenous tribes in that area. The second set of Indigenous names could not be vetted in time with tribal leaders, and so only 2 sets of names were submitted to the IAU.

The U.S. Naming Committee identified many ways in which their procedures should be improved, including but not limited to: 1) work closely with Indigenous institutions from the

\footnotetext{
12 Brandt CB (2008). Discursive geographies in science: space, identity, and scientific discourse among indigenous women in higher education. Cultural Studies of Science Education 3, 703-730 (link).

${ }_{13}$ McMahon TR et al. (2018). Cultivating Native American scientists: an application of an Indigenous model to an undergraduate research experience. Cultural Studies of Science Education 14, 77-110 (link).

${ }^{14}$ See aises.org, sacnas.org, indigenouseducation.org, illuminatives.org, and imiloahawaii.org, respectively.

${ }^{15}$ See www.nameexoworlds.iau.org

${ }^{16}$ Mamajek EE et al. (2018). Division C / Working Group Star Names Triennial Report, IAU (link).
} 
beginning to implement a process for solicitation; 2 ) invite Indigenous leaders to the naming committee, the review committee, and in the design of the contest; 3 ) invite participation directly by representatives from all 573 Tribal Nations in the U.S.; 4) ensure that any Indigenous names submitted be only from members of the referenced tribal community; 5 ) work with the winners of the contest to honor the naming in a way consistent with tribal traditions; 6 ) follow up with First Nations that submit names, in order to continue and grow the relationships that come from this exercise.

\subsection{The naming of Arrokoth}

The trans-Neptunian object 2014 MU69, a 36-km (22-mile) aggregate of ice and rock orbiting far beyond Neptune, was discovered in 2014 during a search for objects that could be flyby targets for the New Horizons spacecraft, after its 2015 flyby of Pluto. In March 2018, NASA announced that 2014 MU69 would be the New Horizons target, and it was given a temporary, unofficial nickname. ${ }^{17}$ The flyby took place on 1 January 2019.

The discoverers decided to use the word Arrokoth as the official name of this object, choosing a word meaning "sky" that is derived from the language of the Powhatan people, who were the original inhabitants of the land where the Johns Hopkins University Applied Physics Laboratory (operations center for New Horizons) now sits. At the prompting of NASA officials, elders from the Powhatan-related communities in the mid-Atlantic area were asked and gave permission to use it. At a ceremony at NASA HQ on 12 November 2019, the permanent name of 2014 MU69 was announced as Arrokoth. Many tribal representatives and leaders attended the ceremony, and a Pamunkey tribal elder offered opening and closing blessings.

The story of Arrokoth's naming is mixed, with regard to the goal of moving beyond cultural appropriation. It is progress, in that permission was sought and obtained from relevant cultural leaders before the name was announced. However, it also serves as an illustration of how much work remains, in that NASA and the spacecraft team were not in a relationship with these tribal communities prior to approaching them about the naming of Arrokoth, and thus the naming process cannot truly be described as co-creative with the Indigenous leaders.

Nevertheless, the lead for NASA/SMD's American Indian/Alaska Native Working Group took this opportunity to build relationships with the tribal leaders initiated by the activities of the naming process and ceremony, with permission from NASA's Planetary Science Division. Those efforts are ongoing and productive, but require funding, time, and intentionality for further partnerships. It will also require deliberate action for future nomenclature efforts to include Indigenous communities from the beginning of the decision-making process.

\subsection{The A Hua He Inoa project}

The A Hua He Inoa (AHHI) nomenclature project ${ }^{18}$ is a partnership between the "Imiloa Astronomy Center of Hawai' $i$, the College of Hawaiian Language at the University of Hawai' $i$ at Hilo (UH Hilo), and Hawai'i-based astronomical observatories, which resolves to "weave traditional indigenous practices into the official naming of astronomical discoveries made in Hawai'i." ${ }^{19}$ Indeed, the name A Hua He Inoa itself means "calling forth a name." The working

\footnotetext{
${ }^{17}$ That nickname, Ultima Thule, led to controversy of its own, but that is beyond the scope of the present paper.

${ }^{18} \mathrm{See}$ https://imiloahawaii.org/news/a-hua-he-inoa-8e3ax

${ }^{19}$ Kimura K et al. (2019). A Hua He Inoa: Hawaiian culture-based celestial naming. Bull. Am. Astron. Soc. 51, 135 (link).
} 
group for $\mathrm{AHHI}$ is made up of people with diverse backgrounds and perspectives, including experts in the Hawaiian language, K-12 educators, astronomers who work at Hawaiian observatories, and community leaders who manage the Maunakea science reserve, the land on which the massive telescopes were built.

On 19 October 2017, UH's Pan-STARRS observatory on Haleakalā, Maui discovered a new object, later determined to be the first detected interstellar object. In coordination with AHHI, this object was named 'Oumuamua, which means "a scout or messenger sent to check things out and quickly depart" in the Hawaiian language. ${ }^{19}$ This name, which was quickly approved by the IAU, garnered attention in the news media and is popular with scientists and the general public alike. We see this as a successful integration of Indigenous nomenclature and science.

Shortly after the naming of 'Oumuamua, a pilot program was conducted in October 2018 between $\mathrm{AHHI}$ and high school and undergraduate students from Maui and Hawai' $\mathrm{i}$, the two islands that host telescopes. While visiting the telescopes and engaging directly with astronomers, the students learned about two recently discovered asteroids and eventually named them. The name of Kamo'oalewa (formerly $2016 \mathrm{HO}$ ) comes from a traditional Hawaiian chant about the origins of the universe called the Kumulipo, and Ka'epaoka'āwela (formerly 2015 BZ509) means "the mischievous opposite-moving companion of Jupiter." ${ }^{19}$ The students responded very positively to the experience, commenting that through this program, they were able to see the link between their ancestral knowledge and modern science.

\section{Recommendations}

For our recommendations, we address three overlapping entities: NASA, the IAU, and the planetary community overall. The IAU is the consensus arbiter of planetary nomenclature, so better policies implemented by the IAU would have far-reaching positive consequences. In the U.S., NASA has the opportunity to implement influential proactive policies for suggesting names to the IAU because, as NASA funds spacecraft and ground-based observations that discover planetary objects and features that then need naming, NASA then can and does coordinate with discoverers on the resulting suggested names. Finally, institutions are responsive to their membership, so all researchers (and especially those involved in discovering and naming planetary objects and features) have a role to play in implementing better procedures and engagement.

For purposes of the Planetary2023 Decadal Survey, the most actionable recommendations are $B$ and $C$ below. We request that the Decadal Survey articulate a priority for federal agencies to build relationships as described below.

\section{A) The planetary community should question the notion that discoverers "own" the right} to name planetary objects or features. Science is a collaborative endeavor. Scientists generally work with resources obtained via public funds and should see themselves as representing all humankind. The act of naming their discoveries, thus, should also be a collaborative endeavor. Furthermore, names themselves are cultural property, reflecting and expressing the hearts of the people who created them, and Western scientists must stop appropriating names from other cultures without consent and participation. ${ }^{20}$ Because

\footnotetext{
${ }^{20}$ Improved diversity in the planetary workforce does address this problem somewhat, inasmuch as discovery teams would be more likely to contain members who can legitimately draw from a wider range of cultural heritage.
} 
reverting to a blatant focus on industrialized cultures for nomenclature is also not an acceptable alternative, co-creation with Indigenous communities is the only tenable option.

B) The planetary community and NASA should build stronger relationships with Indigenous communities, based on equality and cooperation, laying a foundation for co-creation of planetary nomenclature. It is impossible to implement responsible co-creation of planetary nomenclature without a pre-existing foundation of intentional relationships with Indigenous communities. Umbrella organizations such as AISES and SACNAS and IEI (and similar groups around the world) are a good place to start. These relationships should have an open-ended focus on co-creation in scientific endeavors, ${ }^{8}$ and will carry the benefit of being already in place when co-creation of nomenclature is called for.

C) The IAU and NASA should work with a diverse array of Indigenous community leaders to establish a procedure for responsible use of cultural property in planetary nomenclature. We will not specify here the form that this procedure should take, though we do commend initiatives such as AHHI as a model (see Section 3.3). Procedures that proactively avoid cultural appropriation should be worked out organically with Indigenous leaders, as a result of the relationships recommended above. We recommend working with Indigenous leaders to potentially rethink the paradigm of contests or competitions as a framework for selecting names, and to co-conceive with them how to ensure the need to give names to celestial bodies as a co-creative endeavor and as an opportunity for their youth. 